\title{
How is a sensory stimulus represented in ongoing dynamics in the barrel cortex?
}

\author{
Elena Phoka ${ }^{1 *}$, Mark Wildie$^{1}$, Rasmus S Petersen ${ }^{2}$, Mauricio Barahona', Simon R Schultz ${ }^{1}$ \\ From Nineteenth Annual Computational Neuroscience Meeting: CNS*2010 \\ San Antonio, TX, USA. 24-30 July 2010
}

There is an emerging view that the spontaneous activity of the brain does not merely reflect stochastic network fluctuations and internal noise sources, but rather represents the underlying structure [1] or state [2] of the brain. To examine this hypothesis, we studied how a sensory stimulus interacts with the brain's spontaneous activity. Is the spontaneous activity following a sensoryevoked period different from the spontaneous activity before the stimulus input? If so, in what respect does the activity differ - firing rates, or perhaps more subtle aspects of spike correlations or ongoing dynamics? And most importantly, how can such differences be quantified?

As a platform for investigating these questions, we have developed a biologically-inspired model of a rodent barrel column, consisting of approximately 4000 neurons (Izhikevich point neuron model, [3]). We model layers 2, 3 and 4 with the connectivity being random and the connection probabilities for each neuron class (layer 2/3: excitatory pyramidal, and inhibitory basket and non-basket; layer 4: excitatory spiny stellate) constrained by data from the physiological literature (from [4] and elsewhere). STDP occurs at the excitatory synapses, with the particular variety of STDP rule used being cell type and layer dependent. Sensory evoked activity is generated by direct input to layer 4 neurons from a Linear-Nonlinear-Poisson (LNP) model of thalamic nucleus VPm derived from experimentally recorded thalamic transfer functions [5].

We find that the sensory stimulation protocol in the model induces long-lasting changes in synaptic weights in the post-stimulus spontaneous epoch, with substantial heterogeneity amongst cell types and layers. Average neuronal firing rates, in contrast, are essentially unchanged. We are currently investigating how the pattern of synaptic weight changes alters the ongoing dynamical structure of activity. A particular advantage of the modeling approach used here is that we have direct access to the synaptic weight dynamics - which is not feasible with current experimental technology. This provides a means to test hypotheses about the computational role of ongoing cortical dynamics.

\section{Acknowledgements}

This work was supported by BBSRC.

\section{Author details}

${ }^{1}$ Department of Bioengineering, Imperial College London, London, SW7 2AZ, UK. ${ }^{2}$ Faculty of Life Sciences, University of Manchester, Manchester, M60 1QD, UK.

Published: 20 July 2010

\section{References}

1. Kenet T, Bibitchkov D, Tsodyks M, Grivald A, Arieli A: Spontaneously emerging cortical representations of visual attributes. Nature 2003, 425:954-956.

2. Haider B, McCormick DA: Rapid neocortical dynamics: cellular and network mechanisms. Neuron 2009, 62:171-189.

3. Izhikevich EM, Edelman GM: A large-scale model of mammalian thalamocortical systems. PNAS 2008, 105:3593-3598.

4. Lefort S, Tomm C, Sarria FJ-C, Petersen CCH: The excitatory neuronal network of the $\mathrm{C} 2$ barrel column in mouse primary somatosensory cortex. Neuron 2009, 61:301-316.

5. Petersen RS, Brambilla M, Bale MR, Alenda A, Panzeri S, Montemurro MA, Maravall M: Diverse and temporally precise kinetic feature selectivity in the VPm thalamic nucleus. Neuron 2008, 60:890-903.

doi:10.1186/1471-2202-11-S1-P35

Cite this article as: Phoka et al:: How is a sensory stimulus represented in ongoing dynamics in the barrel cortex?. BMC Neuroscience 2010 11(Suppl 1):P35. 